\title{
Comparison of acceptance and distraction strategies in coping with experimentally induced pain
}

\author{
This article was published in the following Dove Press journal: \\ Journal of Pain Research \\ 17 March 2015 \\ Number of times this article has been viewed
}

\begin{abstract}
Background: This study compared an acceptance-based strategy with a control-based strategy (distraction) in terms of the ability of participants to tolerate a painful stimulus, across two experiments. In addition, participants were either actively encouraged, or not, to link pain tolerance with pursuit of valued goals to examine the impact of pursuing a personally meaningful goal or value on the extent to which pain will be tolerated.

Methods: Participants in experiment $1(n=41)$ and experiment $2(n=52)$ were equally assigned to acceptance or distraction protocols. Further, half the participants in each group generated examples from their own lives in which they had pursued a valued objective, while the other half did not. In experiment 2 , the values focus was enhanced to examine the impact on pain tolerance.
\end{abstract}

Results: There were no significant differences overall between the acceptance and distraction groups on pain tolerance in either experiment. However, in experiment 2, individuals classified as accepting in terms of general coping style and who were assigned to the acceptance strategy showed significantly better pain tolerance than accepting individuals who were in the distraction condition. Across both experiments, those with strong goal-driven values in both protocols were more tolerant of pain. Participants appeared to have more difficulty adhering to acceptance than to distraction as a strategy.

Conclusion: Acceptance may be associated with better tolerance of pain, but may also be more difficult to operationalize than distraction in experimental studies. Matching coping style and coping strategy may be most effective, and enhancement of goal-driven values may assist in pain coping.

Keywords: pain, acceptance, values, coping

\section{Introduction}

Chronic pain is a significant health problem, affecting around $19 \%$ of the population. ${ }^{1}$ Psychological intervention may assist patients to develop more effective strategies for controlling pain experience and coping with pain. ${ }^{2}$ For example, distraction is a widely used method within a cognitive-behavioral pain management framework. It is hypothesized that when attention is engaged elsewhere, one does not attend to pain, thus bringing about a degree of analgesia. ${ }^{3}$ Several clinical research studies support distraction as a pain management technique. ${ }^{4-6}$ However, pain demands attention ${ }^{3}$ and impacts on attentional control, ${ }^{7,8}$ so that sustained attentional management of pain may be difficult or impossible. Furthermore, other research has found that diverting attention away from pain can increase the pain experience..$^{9-12}$ One way of explaining such apparent divergence of findings is that perhaps distraction works in some contexts but
Correspondence: Brian E McGuire

Clinical Psychology Programme, School of Psychology and Centre for Pain Research, National University of Ireland, Galway, Ireland

$\mathrm{Tel}+35391493266$

$\mathrm{Fax}+35391534930$

Email brian.mcguire@nuigalway.ie 
not in others. For example, there is evidence that while it can be effective for low-level or short-term pain ${ }^{13,14}$ it is less so when pain is more intense or longer term. ${ }^{15,16}$

Acceptance and commitment therapy (ACT) ${ }^{17}$ and acceptance-based therapies have received attention as an alternative to methods which attempt to control the pain experience. Acceptance-based therapies, such as ACT, focus on context and functions of psychological events rather than the form and frequency of these events. Acceptance-based multidisciplinary pain management programs have reported positive outcomes, ${ }^{18}$ but the processes affecting efficacious acceptance are not as well understood as they might be. Further empirical exploration of these processes is required. One such process, for example, is valuing.

Within the ACT framework, the concept of "valued action" (that is, an action done in the service of some higher goal or valued end, eg, working to make money for one's family) is central and is inextricably linked with all other ACT processes. ${ }^{17}$ Acceptance, without seeking to proceed in a valued direction, is relatively meaningless. Acting in a valued direction is implicit in many therapeutic approaches, including cognitive behavioral therapy. However, ACT makes this focus explicit, which plays a fundamental role in providing motivation and the rationale for making life changes. One mechanism of acceptance is the concept of "pain willingness". Within the ACT framework, pain willingness refers to an openness to experience something in order to progress in a valued direction. Individuals experiencing willingness would therefore not attempt to manipulate or avoid an experience as they pursue their valued objective. Several authors have shown that pain willingness is a valid and measurable concept, where high levels of pain willingness tends to be associated with better psychological adjustment in chronic pain. ${ }^{19,20}$

Previous research of acceptance in the context of chronic pain has demonstrated that acceptance is related to less pain and disability. ${ }^{21-23}$ Previous experimental studies have compared the effects of acceptance-based and control-based instructions for coping with cold pressor pain ${ }^{19-23}$ and electrical stimulation. ${ }^{24-28}$ Results of these studies demonstrated that participants in the acceptance conditions showed better tolerance of pain.

The concept of pain believability was also of interest in this study. Pain believability has been conceptualized differently in different studies, but essentially involves the extent to which a person acts in accordance with the pain. Individuals who demonstrate high pain believability interpret high levels of pain experienced as a reason to stop an activity. Research supports the idea that ACT produces a decrease in pain believability, ${ }^{25,28}$ that is, individuals engaged in acceptance strategies tolerated pain well despite reporting high levels of pain. In particular, research has found that an acceptance-based protocol was superior to a control-based protocol for increasing pain tolerance, but only for participants experiencing higher levels of pain. ${ }^{26}$

The overall aim of this study was to compare the effect of the coping strategy and an induced values focus on pain tolerance and pain willingness. The study replicated and extended a previous study ${ }^{18}$ by examining whether an increase in pain tolerance and pain willingness can be achieved by linking pain tolerance to personally valued actions. Within the current study, it was hypothesized that an acceptance protocol combined with a "high values" condition would have the greatest impact on tolerance for pain, as ACT theory would posit that individuals who adopt acceptance strategies would pursue valued actions despite the presence of unpleasant stimuli such as pain. Previous studies show some support for this assertion. ${ }^{25,27,29}$

The current study sought to operationalize values in such a manner as to make them more personal and therefore more consistent with ACT theory regarding acting in a personally valued direction. It was hypothesized that individuals following the distraction protocol would report lower pain intensity than those in the acceptance condition, as control strategies directly target and attempt to reduce negative painrelated thoughts, but less pain tolerance and less willingness to continue in the face of pain. It was also hypothesized that those following the acceptance protocol would tolerate more pain, in particular in a high pain context. Finally, the study also sought to examine any interaction effects of the assigned coping strategy combined with individual preferred coping style (ie, preferred level of emotional and cognitive control), since the importance of matching coping strategy and style has recently been highlighted in experimental studies of pain distraction. ${ }^{30,31}$

\section{Experiment I Participants}

Fifty-five volunteers participated in this experiment, including 24 students attending the National University of Ireland, Galway, who were recruited through on-campus advertising. They were a mixture of undergraduates and post-graduates from a range of courses of study. The other participants were 31 individuals who were acquaintances of the experimenter. One volunteer was excluded on the basis of a reported heart condition. Ten were excluded because 
they failed to rate the pain as being above 50 on a visual analog scale (VAS) at the pain calibration phase. Three participants were excluded on the basis that they proceeded through all 15 shocks during the pre-intervention task. The final sample consisted of 41 participants ( 26 females,

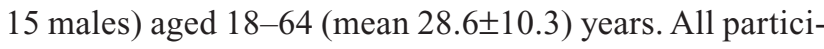
pants received remuneration for participating in the study. Information provided to participants was consistent with recommendations for obtaining informed consent in clinical pain research, ${ }^{32}$ and care was taken to exclude participants if they had circulatory, cardiovascular, or neurological problems, suffered from chronic pain or if they had used analgesics or alcohol in the previous 24 hours, suffered migraine or any medical condition that may be adversely affected by shocks (eg, a metal joint replacement), or had a diagnosed anxiety disorder. ${ }^{33}$

\section{Measures and equipment}

The protocol involved measurement of pain intensity, pain tolerance and pain willingness, coping style, and personal values.

Pain intensity was recorded as the average pain rating reported by the participant on a computer-presented VAS (0 indicating "no pain" to 100 indicating "pain as bad as it could be") in each pain task (outlined in the Procedure section). Pain tolerance was measured by recording the number of shocks the participant chose to receive in each pain task (see Procedure section) and the number of shocks participants endured after reporting high levels of pain (defined as a rating of 80 or over on the VAS).

Pain coping style was measured using the nine-item version of the Acceptance and Action Questionnaire (AAQ). ${ }^{34}$ The measure specifically assesses the need for emotional and cognitive control, avoidance of private events, and inability to take action in the face of negative private events. An aim of using the AAQ in this study was to discern if there were significant differences between the groups in the amount of experiential avoidance they displayed at baseline.

Personal values related to pain were measured using the Valued Living Questionnaire ${ }^{35,36}$ which is designed to assess an individual's consistency of action with their personal values. The Valued Living Questionnaire taps into ten domains often identified as valued domains of living (such as family relationships, partner/intimate relationship, employment, recreation). Individuals are asked to rate, on a scale of 1-10, the importance of each domain and then how consistently they have lived in accordance with their values over the previous week.

\section{Procedure}

The study received ethical approval from the host university (National University of Ireland, Galway) and all participants provided written informed consent. Participants were randomly assigned to one of four conditions involving two coping strategies, ie, acceptance of pain and distraction from pain, and either high or low values, where the participant was either asked to recall a time they had pursued a personally valued goal in the face of obstacles ("high values") or a time they had chosen not to pursue a personally valued goal in the face of obstacles in order to avoid difficult consequences ("low values"; see further details below). Thus, the four conditions were acceptance-high value, acceptance-low value, distraction-high value, and distraction-low value. Two dependent measures of shock tolerance and pain self-report were measured at pre-intervention and post-intervention. The experimenter was blind to allocation. Figure 1 illustrates the sequence of stages in the protocol used in experiment 1.

\section{Pain calibration}

A noxious electrical stimulation was delivered using electrodes attached to the participant's forearm. A button-press attached to the machine was used by the experimenter

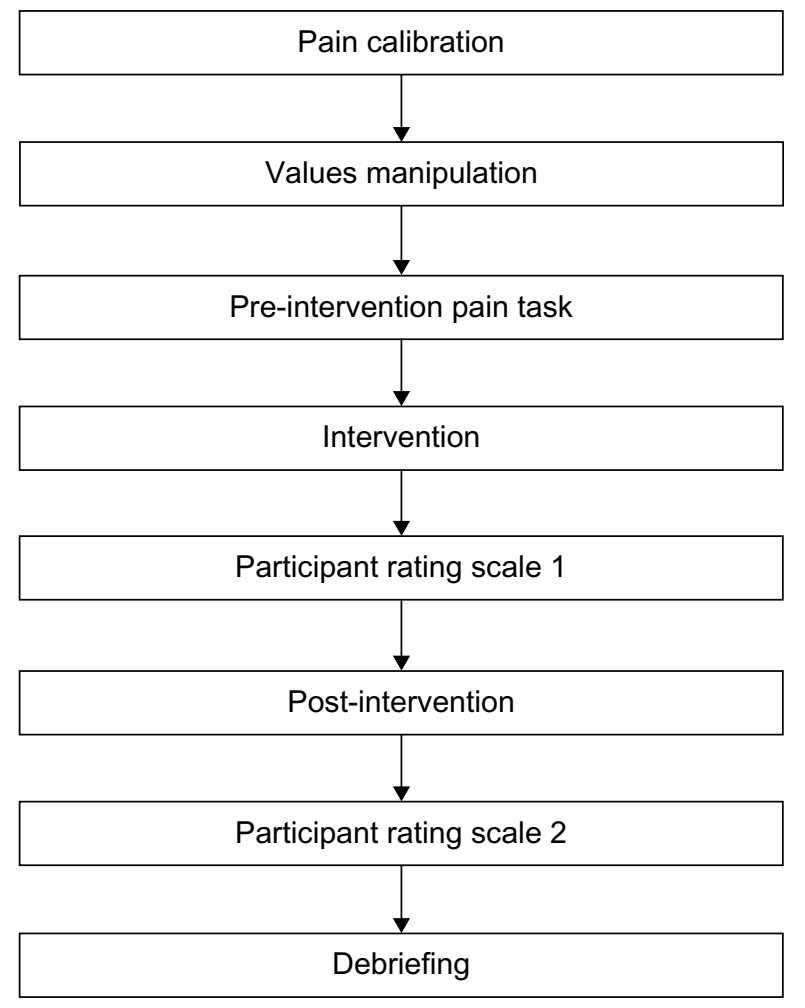

Figure I The sequence of stages in the protocol used in experiment I. The sequence in experiment 2 was identical except that the order of presentation of the values manipulation and pre-intervention pain task stages was reversed. 
to manipulate the duration of the shock. The purpose of this phase was to expose the participant to a maximum of 12 shocks until they rated the pain as being at least half way along a computer-presented VAS pain rating scale from 0 ("no pain") to 100 ("pain as bad as it could be"). Before each shock, two on-screen command buttons appeared on the computer screen, one with the caption "click here to terminate the experiment" and the other with the caption "click here to receive a shock and continue". If the participant chose to end their participation in the experiment, they were thanked and debriefed. If the participant chose to continue, they were exposed to a brief shock and requested to rate their perception of pain on the VAS. This process continued until the participant either chose to discontinue the experiment or until the pain of the shock was rated as above 50 on the VAS. In the latter case, the voltage corresponding to this rating was then set as the stimulation voltage level used for the remainder of the experiment.

\section{Values manipulation}

After the pain calibration stage, participants took part in a values induction exercise. The experimenter left the room while the participant watched six video excerpts. The excerpts were presented by a female therapist who asked participants to imagine themselves in common life situations that involved difficult choices and challenges. This exercise aimed to tap into common valued areas of life. Participants were asked to imagine themselves in each of the presented scenarios in which they were struggling with a decision about whether to pursue their values despite the emotional or physical pain this might cause. Participants received either "high" or "low" value instructions depending on which condition they had been assigned to. Participants in the high value condition were asked to imagine that they chose to make the difficult decision to follow their values despite the pain involved. Participants in the low value condition were asked to imagine that they chose to avoid the difficult consequences and not act in their valued direction.

After presentation of common life situations, participants were asked to generate similar experiences from their own life where there was something they really wanted, and in order to get it, they had to do something that involved experiencing uncomfortable emotions, thoughts, or physical sensations. In the high value condition, participants were asked for an example in which they chose to pursue the action that produced the discomfort, and in the low value condition participants were asked for an example of a time they chose not to pursue the desired objective. The purpose of this was to "prime" the participant prior to commencement of the pain tolerance task.

\section{Pre-intervention pain task}

During this stage, participants performed a cognitive matching task involving a sample stimulus (a number), which appeared at the top of the screen and three comparison stimuli which appeared in a line along the bottom of the screen. The participant was required to identify the matching stimulus. When the participant correctly completed this task, the word "correct" appeared on the screen. If $s /$ he responded incorrectly, nothing appeared on the screen and the computer presented the next matching task. Correct responses were additionally reinforced after every nine correct responses on average (ie, a variable ratio 9 schedule) with visually presented tokens. The tokens were presented to participants in order to make the task intrinsically rewarding. The tokens were presented as part of the matching task both before and after the intervention.

On a variable ratio 11 schedule, a red cross appeared on the screen, indicating that a shock was imminent. Participants were required to make a choice to end the task, or to be shocked and continue. This choice was presented on the screen. If the participant chose to receive a shock they received a brief shock and were asked to rate its painfulness. Self-reported pain ratings were made on the same computerpresented VAS as had been used during the calibration phase. During the pre-intervention cognitive matching task, the voltage level remained at the level set during the pain calibration stage, while the frequency and duration of shocks was incrementally increased across shocks according to a standardized schedule. A maximum of 15 shocks was delivered at this stage. If a participant received all 15 shocks, they were considered to have reached a ceiling, were excluded from the rest of the study, and thanked and debriefed. If, however, they opted to end the pre-intervention task earlier than 15 shocks, they then proceeded to the intervention phase.

\section{Intervention}

Prior to initiating the intervention phase, the experimenter detached the electrodes from the participant's arm. Participants were presented with either pain acceptance-based or distraction-based intervention instructions depending on the condition to which they had been assigned. In the acceptance conditions, participants were given metaphors and experiential exercises derived from ACT designed to teach them that the best way to deal with pain-related thoughts and feelings is to accept them in the context of whatever action is being 
taken. ${ }^{25}$ In the distraction condition, participants were given metaphors and experiential exercises designed to teach them that the best way to deal with pain-related thoughts and feelings is to distract themselves from them by imagining pleasant images. Excerpts from each of the two conditions were as similar as possible in content and word count except for the critical elements (acceptance versus distraction). The intervention scripts, as with the values scripts, were presented on screen by a therapist via pre-recorded, computer-based video. The experimenter was blind to the protocol assigned to each participant. A fidelity check was included whereby participants were asked to summarize, in their own words, the content of video excerpts explaining either the acceptance or distraction strategy in order to demonstrate that they understood the intervention instructions.

\section{Participant rating scale I}

This measure was administered after the intervention video instruction. It required participants to rate on a seven-point Likert scale how useful they felt the strategy would be and how difficult it might be to employ.

\section{Post-intervention pain task}

In this stage, participants again engaged in the cognitive matching task involving the same ratio of shocks as in the pre-intervention phase. Before receiving a shock during this task, however, a video clip reminding participants of the appropriate strategy was shown. After listening to the reminder, the participant had the choice to end the experiment or to continue with the task and receive the shock.

\section{Participant rating scale 2}

After finishing the post-intervention pain task, each participant was asked to rate on a seven-point Likert scale how useful and difficult they had found the intervention strategy during the pain task. They were also asked to record how often they had used the strategy and to record in their own words exactly what strategy they had been employing to cope with the pain.

\section{Debriefing}

At the end of the protocol, all participants were thanked for their participation, and received a debriefing information sheet and their payment.

\section{Analyses}

Analysis of main and interaction effects of strategy (acceptance versus distraction), value (high versus low), and time (pre-intervention $\times$ post-intervention) on the dependent variable of pain tolerance was conducted first with follow-up analysis focused on those who endured relatively high levels of pain. Next, analysis of the same variables on the dependent variable (DV) of self-reported pain was carried out. This included analysis of the possible effect of coping style (accepting or avoidant) as measured using the AAQ on this variable.

For the next stage of analysis, fidelity checks were conducted to determine the extent to which particular intervention strategies were utilized. Participant rating scale 2 required participants to summarize the strategy they were using to cope with the post-intervention pain task. For the purpose of analysis, participants' responses were rated by eight independent evaluators, familiar with the interventions used, for the extent to which they reflected an acceptance or distraction strategy. Ratings of participants' strategy use by each evaluator were correlated with each of the other seven evaluators. Strategy summary ratings were coded so that they were expressed in terms of difference from the maximum strategy adherence, ie, how different the strategy described was from a score of maximum adherence with the assigned strategy. Participant understanding was also compared across conditions. Two independent evaluators rated participants' understanding of the strategy described in the video clip.

Analysis of the usefulness and frequency of use of particular strategies was next, followed finally by therapist believability with respect to strategies. The believability of the therapist delivering the intervention was assessed by two independent evaluators to ensure there were no differences in therapist credibility across the video excerpts. Each video excerpt was rated for the believability of the person delivering the message from 0 (not at all believable) to 10 (highly believable).

\section{Results}

\section{Pain tolerance}

A $2 \times 2 \times 2$ mixed analysis of variance design was employed to examine interactions between strategy (acceptance versus distraction), value (high versus low), and the within-subject variable of time (pre- versus post-intervention) upon pain tolerance. No main or interaction effects were found for strategy or values. There was a tendency, which did not reach significance, for participants to take more shocks in the post-intervention pain task (mean 6.22 \pm 4.20 ) than in the pre-intervention pain task (mean 5.12 $\pm 3.76 ; F(1,37)=3.123$, $P=0.09, \eta^{2}=0.08$, see Table 1$)$. After this, to examine pain tolerance, we compared 12 participants, seven in the 
Table I Results of a three-way mixed analysis of variance; the effect of strategy and value on shock tolerance during pre-intervention and post-intervention matching tasks in experiment I

\begin{tabular}{lllll}
\hline Source & $\mathbf{d f}$ & $\boldsymbol{F}$ & $\eta^{2}$ & $\boldsymbol{P}$-value \\
\hline Between subjects & & & & \\
Strategy & $\mathrm{I}$ & 4.12 & 0.01 & 0.53 \\
Value & $\mathrm{I}$ & 0.12 & 0.00 & 0.92 \\
Strategy $\times$ value & $\mathrm{I}$ & 4.12 & 0.01 & 0.53 \\
Within-group error & 37 & $(25.77)$ & & \\
Within subjects & & & & \\
Time & $\mathrm{I}$ & 3.12 & 0.08 & 0.09 \\
Time $\times$ strategy & $\mathrm{I}$ & 0.00 & 0.00 & 0.99 \\
Time $\times$ value & $\mathrm{I}$ & 0.11 & 0.00 & 0.75 \\
Time $\times$ strategy $\times$ value & $\mathrm{I}$ & 0.10 & 0.00 & 0.75 \\
Within-group error & 37 & $(7.90)$ & & \\
\hline Note: Valus &
\end{tabular}

Note: Values enclosed in parentheses represent mean square errors.

acceptance condition and five in the control condition, who endured further shocks after providing a pain rating of 80 or more. A Mann-Whitney $U$ test was performed to investigate if participants in the acceptance condition tolerated more shocks, and there was no significant difference.

\section{Self-reported pain}

To examine self-reported pain (VAS score), a $2 \times 2 \times 2$ mixed analysis of variance was similarly conducted with pain self-report as the dependent variable. Analysis of variance showed no main or interaction effects for either strategy or values (see Table 2).

We then looked at scores on the AAQ, which indicated how accepting or avoiding a participant was, and conducted a two-way between-subjects analysis of variance with AAQ score (15 highest versus 15 lowest scoring) and strategy as the independent variables and pain difference (ie, between the pre-intervention and post-intervention pain tasks) as the dependent variable. No significant main effect for AAQ scores was found and no significant interaction effect was found between AAQ score and strategy.

\section{Fidelity checks}

Analysis of the extent to which the respective intervention strategies were utilized found a significant difference between conditions such that participants in the acceptance conditions did not appear to adhere as well to the assigned strategy (mean rank 27.16) as those in the distraction condition (mean rank 14.38; $U=71.00, \mathrm{n}=40, P<0.001)$.

Two independent evaluators rated participants' understanding of the strategy described in the video clip. Expert raters rated participants' understanding of, and adherence to, either the acceptance-based or control-based strategy
Table 2 Results of three-way mixed analysis of variance; the effect of strategy and value on self-reported pain during pre-intervention and post-intervention matching tasks in experiment I

\begin{tabular}{lllll}
\hline Source & $\mathbf{d f}$ & $\boldsymbol{F}$ & $\eta^{2}$ & $\boldsymbol{P}$-value \\
\hline Between subjects & & & & \\
Strategy & $\mathrm{I}$ & 2.75 & 0.08 & $0.1 \mathrm{I}$ \\
Value & $\mathrm{I}$ & 0.45 & $0.0 \mathrm{I}$ & $0.5 \mathrm{I}$ \\
Strategy $\times$ value & $\mathrm{I}$ & $\mathrm{I} .66$ & 0.05 & $0.2 \mathrm{I}$ \\
Within-group error & 34 & $(627.02)$ & & \\
Within subjects & & & & \\
Time & $\mathrm{I}$ & 0.49 & $0.0 \mathrm{I}$ & 0.49 \\
Time $\times$ strategy & $\mathrm{I}$ & 30.46 & 0.09 & 0.07 \\
Time $\times$ value & $\mathrm{I}$ & 0.30 & 0.01 & 0.59 \\
Time $\times$ strategy $\times$ value & $\mathrm{I}$ & $0.0 \mathrm{I}$ & 0.00 & 0.94 \\
Within-group error & 34 & $(\mathrm{I} 03.2 \mathrm{I})$ & & \\
\hline
\end{tabular}

Note: Values enclosed in parentheses represent mean square errors.

to which they were assigned using a five-point Likert scale where a score of 1 represented very low understanding or adherence. Ratings of participant understanding of video excerpts by each of the two raters were highly correlated. Participants in the distraction condition had a higher mean understanding rating than those in the acceptance condition, although the difference was not statistically significant.

\section{Ratings of usefulness of strategy}

There was no difference between groups in their ratings of the usefulness of the strategies (see Table 3). There was a significant difference on the variable of difficulty between high-value and low-value groups $(U=113.50, P=0.03)$. The participants in the low value conditions rated the strategy as more difficult to use (mean rank 24.03) than those in the high value conditions (mean rank 16.18). There was also a significant difference in the reported frequency of use of strategy; participants in the distraction condition reported using the strategy more often (mean rank 24.10) than those in the acceptance condition (mean rank 15.68; $U=108.00$, $P=0.02$ ).

\section{Believability of therapist}

The believability of the therapist delivering the intervention was assessed by two independent evaluators to ensure there were no differences in therapist credibility across the video excerpts. Each video excerpt was rated for the believability of the person delivering the message from 0 (not at all believable) to 10 (highly believable). The results were not significant ( $U=5.00, P=0.10$ and $U=17.00, P=0.85$ in each case, respectively), indicating similarity in therapist believability across video excerpts. 
Table 3 Mean scores and standard deviations (in parentheses) for participants in each condition on each variable in participant rating scales I and 2 (usefulness and difficulty of the strategies) in experiment I

\begin{tabular}{|c|c|c|c|c|c|}
\hline & \multicolumn{2}{|c|}{ Participant rating scale I } & \multicolumn{3}{|c|}{ Participant rating scale 2} \\
\hline & Usefulness & Difficulty & Usefulness & Difficulty & Frequency \\
\hline Acceptance-high value & $6.60(1.95)$ & $4.60(2.46)$ & $6.50(2.37)$ & $4.00(2.16)$ & $6.00(2.45)$ \\
\hline Acceptance-low value & $5.90(2.03)$ & $6.20(1.69)$ & $6.44(2.5 \mathrm{I})$ & $6.33(2.06)$ & $5.67(2.60)$ \\
\hline Control-high value & $7.80(1.8 I)$ & $5.50(3.14)$ & $6.50(2.99)$ & $4.10(3.54)$ & $6.60(4.60)$ \\
\hline Control-low value & $5.73(2.6 \mathrm{I})$ & $5.64(3.17)$ & $6.80(2.78)$ & $5.80(2.86)$ & $8.60(2.12)$ \\
\hline
\end{tabular}

\section{Discussion}

The following issues were identified as limitations in experiment 1 and led to changes in experiment 2 .

\section{Values manipulation}

This section of the protocol appeared to be causing some confusion. A number of participants appeared to fail to link the values manipulation with the rest of the experiment. This was illustrated by two observations. During the intervention phase of the protocol, participants were asked to access the personal example they had provided during the values exercise. It was evident from the intervention video summary sheets completed at this stage that many participants failed to remember the example or misunderstood the reference. Second, a number of participants asked questions on terminating the experiment, which also indicated that they had misunderstood or failed to access the personal values example. It was surmised that the time delay between producing this example and being asked to retrieve it toward the end of the intervention may have been too long.

\section{Reason participants chose to terminate the post-intervention pain task}

Some participants commented that there were other factors in addition to the experience of pain, or indeed unrelated to the experience of pain, which caused them to choose to terminate. There was a concern that if participants were terminating their participation due to factors other than the pain, the results of the experiment may be uninterpretable.

Table 4 Results of a two-way analysis of covariance; the effect of strategy and value on shock tolerance during pre-intervention and post-intervention matching tasks

\begin{tabular}{lllll}
\hline Source & $\mathbf{d f}$ & $\boldsymbol{F}$ & $\eta^{2}$ & P-value \\
\hline Between subjects & & & & \\
Strategy & $\mathrm{I}$ & 0.802 & 0.02 & 0.38 \\
Value & $\mathrm{I}$ & $\mathrm{I} .30$ & 0.03 & 0.26 \\
Strategy $\times$ value & $\mathrm{I}$ & 0.04 & 0.00 & 0.85 \\
Error & 47 & $(19.73)$ & & \\
\hline
\end{tabular}

Note: Values enclosed in parentheses represent mean square errors.

\section{Electrical stimulation}

Some participants in experiment 1 commented that they felt the level of shock had varied between the pre-intervention and post-intervention pain tasks. Most of these participants indicated perceiving the shock as less painful in the postintervention pain task. The detaching of the electrodes from the participant's arm between the pre-intervention and post-intervention pain tasks may have been responsible for this observation. It is possible that the electrodes were not returned to an exactly similar position on the participant's arm, thereby causing a variation in pain perception. The issues outlined above led to the conducting of a second experiment with changes made to the experimental protocol.

\section{Experiment 2 \\ Participants}

Fifty-nine participants were recruited by advertising in the host university. Three volunteers were excluded on the basis of the medical exclusion criteria. Four participants were excluded on the basis that they proceeded through all 15 shocks during the pre-intervention task. The final sample consisted of 52 participants ( 39 females, 13 males) aged

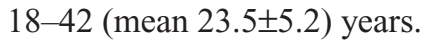

\section{Measures and equipment}

The measures and equipment were similar to those in experiment 1 with one exception. An additional question was included on participant rating scale 2 about the main factor that caused participants to discontinue the post-intervention pain task.

\section{Procedure}

The conditions under which the experiment took place and the preliminary procedures were exactly similar to experiment 1 (see Figure 1). However, the sequence of the protocol was changed slightly in experiment 2 (also in Figure 1).

On the pre-intervention pain task, the protocol was altered so that the electrodes were not removed from the participant's arm. The cable connecting the electrodes from the electric 
shock simulator to the participant's arm was instead disconnected from the machine, leaving the participant free to walk around the room with the electrodes remaining attached to their arm.

The values intervention was altered so that all participants received the values intervention after the pre-intervention matching task instead of prior to it. The change in the protocol sequence from experiment 1 aimed to decrease the period of time between the values manipulation and the intervention video excerpts, so that the values manipulation would be more closely linked to the coping task.

Additional instructions regarding the value of the experiment were provided during the values video excerpts, and the intervention and post-intervention phases of the experiment for individuals in the high value conditions only. The additional instructions emphasized that the pain task is similar to the dilemma of a chronic pain sufferer who must carry on with life activities despite the pain, and that the strategy provided might be one used by chronic pain sufferers and participation in the study will help contribute to the development of more effective therapeutic interventions for individuals suffering from chronic pain.

\section{Analyses}

First, a two-way analysis of variance (acceptance versus control, high value versus low value) with pre-intervention shock tolerance as the DV was performed as a randomization check. After this, a two-way analysis of covariance with pre-intervention shock tolerance as the covariate and postintervention shock tolerance as the DV was employed.

To examine self-reported pain tolerance and pain intensity, a three-way mixed analysis of variance was conducted which included strategy (acceptance $\times$ distraction), value (high $\times$ low), and the within-subject variable of time (pre- versus postintervention). Analysis of variance was also used to examine the possible effect on this variable of coping style (accepting or avoidant, gauged using the AAQ).

Data from participants who identified pain as the primary reason for ending the post-intervention pain task were separated from the main data and analyzed in order to investigate if there were any main or interaction effects for strategy and value on pain tolerance and self-report.

Thereafter, analyses were conducted on data concerning: the extent to which particular intervention strategies were used; participant understanding of strategy; usefulness of strategy; frequency of use of strategy; and believability of the therapist. These analyses were similar to those conducted in experiment 1 .

\section{Results}

\section{Pain tolerance}

The two-way analysis of variance (acceptance $\times$ control, value $[$ high $\times$ low]) with pre-intervention pain tolerance as the DV conducted as a randomization check revealed a significant strategy and value interaction. For the analysis of postintervention pain tolerance, therefore, a two-way analysis of covariance with pre-intervention shock tolerance as the covariate was employed to control for the latter. This showed no main or interaction effects for either strategy or values. We also compared those in the acceptance group $(n=7)$ with those in the distraction condition $(n=6)$ who endured further shocks after providing a pain rating of 80 or more. A MannWhitney $U$ test revealed no difference according to strategy in the number of shocks received $(U=18.5, P=0.73)$.

\section{Self-reported pain}

The three-way mixed analysis of variance with strategy (acceptance $\times$ distraction), value (high $\times$ low), and the withinsubject variable of time (pre- versus post-intervention; see Table 5) showed no significant main or interaction effects for either strategy or values.

We then looked at scores on the AAQ, which indicated how accepting or avoidant a participant was, and conducted a two-way between-subjects analysis of variance with AAQ score and strategy as the independent variables and pain difference (between the pre-intervention and postintervention pain tasks) as the dependent variable. A main interaction effect was found for AAQ category and strategy $\left(F(3,26)=4.80, P=0.04, \eta^{2}=0.16\right)$. An independent Student's $t$-test with difference in pain tolerance as the DV revealed that accepting individuals in the acceptance strategy condition (mean 5.5 \pm 5.29 ) showed a significantly higher increase in

Table 5 Results of three-way mixed analysis of variance; the effect of strategy and value on self-reported pain intensity during preintervention and post-intervention matching tasks in experiment 2

\begin{tabular}{lllll}
\hline Source & $\mathbf{d f}$ & $\boldsymbol{F}$ & $\eta^{2}$ & $\boldsymbol{P}$ \\
\hline Between subjects & & & & \\
Strategy & $\mathrm{I}$ & 0.05 & 0.01 & 0.82 \\
Value & $\mathrm{I}$ & 0.23 & 0.01 & 0.63 \\
Strategy $\times$ value & $\mathrm{I}$ & 3.93 & 0.09 & 0.06 \\
Within-group error & 39 & $(822.96)$ & & \\
Within subjects & & & & \\
Time & $\mathrm{I}$ & 0.00 & 0.00 & 0.95 \\
Time $\times$ strategy & $\mathrm{I}$ & 0.18 & 0.01 & 0.68 \\
Time $\times$ value & $\mathrm{I}$ & 0.0 .05 & 0.00 & 0.83 \\
Time $\times$ strategy $\times$ value & $\mathrm{I}$ & 0.28 & 0.01 & 0.60 \\
Within-group error & 38 & $(55.92)$ & & \\
\hline Note: Values & & & & \\
\hline
\end{tabular}

Note: Values enclosed in parentheses represent mean square errors. 
shock tolerance than accepting individuals in the distraction condition (mean $0.71 \pm 2.49 ; t(13)=2.18, P=0.05)$. An additional analysis was carried out to examine if those participants in the acceptance condition with the highest scores on the AAQ would be significantly different from those with lower AAQ scores in how they adhered to the acceptance strategy. Results of this analysis revealed a significant difference between groups ( $U=9.00, \mathrm{n}=14, P=0.05$ ). Participants who were classified by the AAQ as more accepting adhered more successfully to the acceptance strategy (mean rank 9.38) than those who had been classified by the AAQ as avoiding (mean rank 5.00).

\section{Analysis of reasons for stopping}

Where relevant, participants were asked to identify the reason they chose to end the post-intervention identity-matching pain task before completion (15 shocks). Participants' main reasons for choosing to end the post-intervention identity-matching task were intolerance of pain (56\%), boredom/impatience (22\%), lack of incentive (9\%), anxiety $(6 \%)$, and goal reached $(6 \%)$. Data from participants who identified pain as the primary reason for ending the post-intervention pain task were separated from the main data and analyzed in order to investigate if there were any main or interaction effects for strategy and value on pain tolerance and self-report. Main effects on the dependent variables of shock tolerance and pain self-report might be expected for participants who were truly struggling with pain, as was intended based on the design of the study. A Kruskal-Wallis test examining the dependent variable of shock difference (difference between shock tolerance in the pre-intervention and post-intervention pain task) showed no significant difference between each of the four conditions $\left(\chi^{2}(3, \mathrm{n}=25)=5.70, P=0.13\right)$. A Mann-Whitney $U$ test revealed a significant difference between the high value and low value conditions. Participants in the high value conditions increased their shock tolerance (mean rank 16.27) significantly more than those in the low value conditions (mean rank 9.46; $U=35.50, P=0.02$ ).

\section{Strategy adherence}

The raters were in agreement (all correlations at the $P<0.001$ level) on strategy use across participants. The results indicated that participants in the acceptance strategy conditions did not adhere to the strategy (mean rank 36.58) to the same extent as those in the distraction conditions (mean rank 16.42; $U=36.00, \mathrm{n}=40, P<0.001)$.

Ratings of participant understanding of video excerpts by each of the two raters were highly correlated. Individuals in the acceptance/high value condition had significantly lower ratings of understanding (mean rank 8.63) than participants in the distraction/high value condition (mean rank 14.45; $U=25.50, P=0.02)$.

\section{Ratings of usefulness of strategy}

There was no difference between groups in their ratings of the usefulness of the strategies (see Table 6). There was a significant difference for the variable of difficulty between groups; participants in the distraction/high value condition rated the strategy as more difficult to use (mean rank 16.14) than those in the acceptance/high value condition (mean rank 9.42; $U=31.50, P=0.02$ ). A Mann-Whitney $U$ test also revealed a significant difference for the variable of frequency of strategy use $(U=185.50, P=0.02)$. Similar to experiment 1 , participants in the distraction conditions reported using the strategy more often (mean rank 29.93) than those in the acceptance conditions (mean rank 20.63).

\section{Therapist believability}

Regarding ratings of therapist believability, the results were not significant $(U=5.00, P=0.10$ and $U=17.00, P=0.85$ for acceptance and control conditions, respectively), indicating similarity in therapist believability across video excerpts.

\section{Discussion}

This study compared an acceptance-based strategy and a distraction strategy, along with either a high or low personal values protocol, in tolerating painful electrical stimulation. An overview of the findings of the current study is as follows.

Table 6 Mean scores and standard deviations (in parentheses) for participants in each condition on each variable in participant rating scales I and 2 (usefulness and difficulty of the strategies) in experiment 2

\begin{tabular}{|c|c|c|c|c|c|}
\hline & \multicolumn{2}{|c|}{ Participant rating scale I } & \multicolumn{3}{|c|}{ Participant rating scale 2} \\
\hline & Usefulness & Difficulty & Usefulness & Difficulty & Frequency \\
\hline Acceptance-high value & $6.15(2.48)$ & $4.38(2.33)$ & $6.23(2.24)$ & $4.08(2.84)$ & $6.46(2.54)$ \\
\hline Acceptance-low value & $5.38(2.18)$ & $6.38(2.22)$ & $4.77(2.77)$ & $6.85(2.58)$ & $4.92(3.10)$ \\
\hline Control-high value & $6.23(1.24)$ & $7.46(1.27)$ & $6.45(1.87)$ & $6.91(1.45)$ & $7.91(2.63)$ \\
\hline Control-low value & $6.69(2.14)$ & $6.31(2.12)$ & $6.83(1.34)$ & $6.08(2.15)$ & $7.25(1.87)$ \\
\hline
\end{tabular}


There were no significant differences overall between the acceptance and distraction groups with regard to pain tolerance in either experiment. However, in experiment 2, individuals classified as accepting in terms of general coping style and who were assigned to the acceptance strategy, showed significantly better pain tolerance than accepting individuals who were in the distraction condition. In addition, across both experiments, those with strong goal-driven values in both protocols appeared to be more tolerant of pain. Finally, participants appeared to have more difficulty adhering to acceptance than to distraction as a strategy.

Based on previous research, it was hypothesized that the acceptance rationale combined with the high value condition would have the greatest impact on tolerance for pain. Previous experimental research has suggested that acceptance-based strategies may be more successful than control-based strategies (such as distraction) at facilitating an increase in pain tolerance. ${ }^{25-28}$ This was not found in either experiment. However, there is evidence that those in the acceptance condition did not implement their assigned strategy as well as those in the distraction condition.

A main effect of values on pain tolerance was observed for participants in experiment 2 who identified pain as the primary reason they chose to end the post-intervention pain task. It is suggested that these participants, who were truly struggling with pain, best reflect the aim of producing a difficult choice for participants in relation to continuing (due to the experience of pain) as was intended in the design of the study. Participants in the high value conditions increased their shock tolerance from pre-intervention to post-intervention. Although there was no significant effect for strategy, the influence of value was directionally consistent with the main hypothesis.

It was hypothesized that individuals using the distraction strategy would report less pain than those in the acceptance condition, given that control strategies directly target and attempt to reduce negative pain-related thoughts. Similar to Johnson et al, ${ }^{27}$ this hypothesis was not supported in the current study. Distraction is generally thought to be helpful for dealing with acute pain so the reasons for our findings are unclear. However, some possible explanations are that the distraction technique was not powerful enough, or not powerful enough in the context of a demanding experimental protocol. It is also possible that those utilizing distraction were not well matched in terms of a preference for using distraction to deal with pain. As noted, in experiment 2, we found a difference for individuals classified as accepting by their AAQ scores; accepting individuals in the acceptance conditions increased their shock tolerance from pre-intervention to post-intervention more than did accepting individuals in the distraction condition. This supports the findings of other studies that have also indicated the importance of matching coping strategy with coping style. ${ }^{30,31}$ However, we did not measure preference for avoidance/distraction as a preferred coping response in the presence of pain.

The lack of significant results for strategy is puzzling in light of the moderate or large effect sizes reported in some other studies. ${ }^{25-28}$ A close examination of the adherence measures incorporated into the current study may help to explain the findings. The adherence data suggest that individuals may not have adequately understood the acceptance intervention and may have used a strategy that did not actually reflect acceptance. Perhaps the strongest evidence for this is revealed by data from ratings of participants' summaries of the strategies they used to cope with the pain, which showed that distraction participants were better at using distraction strategies than participants in the acceptance conditions were at reflecting an acceptance stance. Acceptance is a complex strategy involving several aspects or features and having a number of correlates and predictors. ${ }^{19-22}$ This complex psychological process presents challenges for clinical populations, and may be even more challenging for nonclinical populations where the pain experience is limited in time. Acceptance has also been identified as difficult to teach effectively, especially in the absence of a concrete, life-relevant goal. ${ }^{37}$ Our primary interest in conducting this study was to look at different strategies for responding to pain, namely acceptance and control (distraction). However, acceptance in clinical situations tends to be used as a possible way of coping with persistent pain. Our experimental paradigm used an acute pain stimulus and so due caution must be taken in not overinterpreting findings from an experimental acute pain paradigm to the challenge of coping with chronic daily pain. In that context, it is quite possible that the acceptance strategy used by participants in this experiment was not an adequate representation of acceptance as used in clinical pain contexts.

Independent evaluations of participants' summaries of the video interventions also revealed a pattern of lower understanding in the acceptance conditions. Additionally, participants in the distraction conditions in both experiments reported using the strategy more often than participants in the acceptance conditions.

In terms of the role of values, there was a lack of a significant main effect for the manipulation of the values context carried out in experiment 1 and the altered values 
manipulation carried out in experiment 2. There was, however, a significant effect of value on pain tolerance in participants in experiment 2 who identified pain as the primary reason they chose to end the post-intervention pain task. Participants in the high value conditions increased their shock tolerance significantly more than those in the low value conditions. This may suggest that those in the high values condition were motivated to continue with the pain task.

The lack of the expected results for acceptance combined with a high value context could be interpreted as a failure of the values manipulation. It is possible that participants did not value participation in the experiment, as was required in the high value conditions. Some evidence supports this hypothesis. For example, some participants in both experiments decided not to subject themselves to any shock in the pre-intervention pain task. Similarly, $31 \%$ chose to end the post-intervention pain task due to impatience, boredom, or lack of incentive. This indicates that these participants did not value the goal of getting to the end of the experiment. In a helpful review of the role of motivation in attentional aspects of pain, it has been suggested that the degree to which attentional control is successful may well depend on contextual factors such as the characteristics of the pain itself and the characteristics of the goal being pursued. This may be especially important if the pursued activity is directed toward amelioration of the pain, in which case attention may be more likely to be captured by the pain. ${ }^{38}$

Future similar experimental research studies might benefit from incorporating some additional measures in relation to the values dimension. In particular, it may be beneficial to include a Likert scale item requiring participants to indicate how important they perceive it to be that they reach the end of the task or asking participants whether or not they felt the research could offer a valuable contribution, thereby identifying the value they assigned to participation.

An additional factor that may have contributed to the lack of significant results in this study was a possible difficulty with the concept of values as operationalized in the study. The conceptualization of values in this study relates more to short-term "trivial" goals rather than the ACT conceptualization of values that involves defining life directions.

The study has several limitations. From an ACT perspective, a limitation of the pain task was that it was of no obvious value for participants to proceed. There was an altruistic rationale (to help those with chronic pain) provided to those in the high value condition in experiment 2 , but this may not have been personal enough to affect behavior. A suggestion for future research would be to personalize the values manipulation in similar studies. In particular, research might best target specific values where a discrepancy has been identified between importance and pursuit. This is also more likely to resemble more closely the approach taken by clinicians using ACT with those affected by chronic pain.

The sample size of both experiments was relatively small. However, it should be noted that the $n$ value obtained in both the current experiments is comparable with the sample sizes used in similar studies. ${ }^{11,16,17}$

The experiment was somewhat complicated and difficult for participants to navigate. The experimental protocol required high levels of concentration in addition to the ability to switch tasks frequently, work on a computer, listen to and summarize the video-presented protocol in written format, access and write down personal information from their own lives, and engage in physical activity. While the variety of tasks to be navigated undoubtedly made the experiment more interesting, the possibility is increased that participants were unable to fully process and understand what was being asked of them.

There is some concern that the instructions in relation to acceptance in the study were not sufficient. Acceptance is a very complex dynamic construct that involves reorienting perceptions and expectations. It is difficult to teach effectively in an experimental setting, removed from the context of real-life experience. Furthermore, the participants in this study were pain-free and the pain experienced was acute. It is possible that patients with chronic pain might react differently. It also seems likely that the idea of acceptance will make more "sense" for a person with chronic pain in comparison to a person with acute, experimentallyinduced pain.

Future research may consider dividing the protocol into separate sessions conducted on individual days in order to reduce the demands on participants. Additional motivating contexts might also be added, such as introducing an element of competition.

This study may be educational in indicating factors that require further attention from researchers in the context of experimental studies of acceptance. These factors include lack of understanding of acceptance and lack of valuing of participation.

There is evidence suggesting that matching preferred coping strategies with actual coping strategies improves the effectiveness of those strategies with regard to pain threshold and tolerance. ${ }^{24,25}$ Future experimental studies may help refine clinical interventions by discovering which individuals would be most likely to benefit from acceptance-based 
or control-based interventions and in what context (eg, high pain intensity, short-term or long-term pain). Further research is needed to achieve such refinement.

\section{Disclosure}

The authors report no conflicts of interest in this work.

\section{References}

1. Breivik H, Collett B, Ventafridda V, Cohen R, Gallacher D. Survey of chronic pain in Europe: prevalence, impact on daily life, and treatment. Eur J Pain. 2006;10:287-333.

2. Williams AC, Eccleston C, Morley S. Psychological therapies for the management of chronic pain (excluding headache) in adults. Cochrane Database Syst Rev. 2009;2:CD007407.

3. Eccleston C, Crombez G. Pain demands attention: a cognitive-affective model of the interruptive function of pain. Psychol Bull. 1999;125: 356-366.

4. Brewer BW, Karoly P. Effects of attentional focusing on pain perception. Motiv Emot. 1989;13:193-203.

5. Harvey AG, McGuire BE. Suppressing and attending to painrelated thoughts in chronic pain patients. Behav Res Ther. 2000;38: $1117-1124$.

6. Affleck G, Urrows S, Tenne H, Higgins P. Coping with pain from rheumatoid arthritis: patterns and correlates. Pain. 1992;51:221-229.

7. Schoth DE, Nunes VD, Liossi C. Attentional bias towards painrelated information in chronic pain; a meta-analysis of visual-probe investigations. Clin Psychol Rev. 2012;32:13-25.

8. Moriarty O, McGuire BE, Finn DP. The effect of pain and its pharmacological treatment on cognitive function: a review of clinical and pre-clinical studies. Prog Neurobiol. 2011;93:385-404.

9. Goubert L, Crombez G, Eccleston C, Devulder J. Distraction from chronic pain during a pain-inducing activity is associated with greater post-activity pain. Pain. 2004;110:220-227.

10. Keefe FJ, Williams DA. A comparison of coping strategies in chronic pain patients in different age groups. J Gerontol. 1990;45:161-165.

11. Koster EH, Rassin E, Crombez G, Naring GW. The paradoxical effects of suppressing noxious thoughts during imminent threat. Behav Res Ther. 2003;41(9):1113-1120.

12. Cioffi D, Holloway J. Delayed costs of suppressed pain. J Pers Soc Psychol. 1993;64:274-282.

13. Mullen B, Suls J. The effectiveness of attention and rejection as coping styles: a meta-analysis of temporal differences. J Psychosom Res. 1982;26:43-49.

14. Suls J, Fletcher B. The relative efficacy of avoidant and nonavoidant coping strategies: a meta-analysis. Health Psychol. 1985;4:279-288.

15. Ahles TA, Blanchard EB, Leventhal H. Cognitive control of pain: attention to the sensory aspects of the cold pressor stimulus. Cogn Ther Res. 1983;7:159-178.

16. McCaul KD, Haugvedt C. Attention, distraction and cold-pressor pain. J Pers Soc Psychol. 1982;43:154-162.

17. Hayes SC, Strosal K, Wilson KG. Acceptance and Commitment Therapy: An Experiential Approach to Behaviour Change. New York, NY, USA: Guilford; 1999.

18. Veehof MM, Oskam MJ, Schreurs KM, Bohlmeijer ET. Acceptancebased interventions for the treatment of chronic pain: a systematic review and meta-analysis. Pain. 2011;15:533-542.

19. Fish R, McGuire BE, Hogan M, Stewart I, Morrison T. Validation of the Chronic Pain Acceptance Questionnaire (CPAQ) in an Internet sample and development and preliminary validation of the CPAQ-8. Pain. 2010;149:435-443.
20. Fish RA, Hogan MJ, Morrison TG, Stewart I, McGuire BE. Willing and able: a closer look at pain willingness and activity engagement in chronic pain. J Pain. 2013;14:233-245.

21. McCracken LM, Vowles KE, Eccleston C. Acceptance based treatment for persons with complex, long standing chronic pain: a preliminary analysis of treatment outcome in comparison to a waiting phase. Behav Res Ther. 2005;43:1335-1346.

22. McCracken LM, Eccleston C. Coping or acceptance; what to do about chronic pain? Pain. 2003;105:197-204.

23. Dahl J, Wilson KG, Nilsson A. Acceptance and commitment therapy and the treatment of persons at risk of long term disability resulting from stress and pain symptoms: a preliminary randomised trial. Behav Ther. 2004;35:785-802.

24. Korn Z. Effects of acceptance/commitment and cognitive behavioural interventions on pain tolerance. Unpublished doctoral dissertation. New York, NY, USA: Hofstra University; 1997.

25. Hayes SC, Bissett RT, Korn Z, et al. The impact of acceptance versus control rationales on pain tolerance. Psychol Rec. 2001;49:33-47.

26. Gutierrez O, Luciano C, Rodriquez M, Fink BC. Comparison between an acceptance-based and a cognitive-control based protocol for coping with pain. Behav Ther. 2004;35:767-784.

27. Johnson B, Stewart I, Barnes-Holmes D, Barnes-Holmes Y, Luciano C, Wilson K. Doing what you do not want to do to achieve valued outcomes: acceptance and valuing in the face of aversive stimulation. Paper presented at the Behaviour Therapy: Process oriented Experimental Analyses symposium, Annual Conference of the Psychological Society of Ireland, Cork, Ireland, November 6-10, 2004.

28. McMullen J, Barnes-Holmes D, Barnes-Holmes Y, Stewart I, Luciano C, Cochrane A. Acceptance versus distraction: brief instructions, metaphors and exercises in increasing tolerance for self-delivered electric shocks. Behav Res Ther. 2008;46:122-129.

29. Bach P, Hayes SC. The use of acceptance and commitment therapy to prevent the rehospitalisation of psychotic patients: a randomised controlled trial. J Consult Clin Psychol. 2002;70:1129-1139.

30. Forys KL, Dahlquist LM. The influence of preferred coping style and cognitive strategy on laboratory-induced pain. Health Psychol. 2007;26:22-29.

31. Fox L, Walsh JC, O' Gorman D, Ruane N, Carey JJ, Coughlan R, McGuire BE. The differential impact of preferred cognitive coping style on the effectiveness of interventions to reduce pain and anxiety in chronic pain patients. Paper presented at the 31 st annual Stress and Anxiety Research Society conference, NUI Galway. 2010.

32. Cassarett D, Karlawish J, Sankar P, Hirschma, Asch DA. Obtaining informed consent for clinical pain research: patients' concerns and information needs. Pain. 2001;92:71-79.

33. Rhudy JL, Meagher MW. Individual differences in the emotional reaction to shock determine whether hypoalgesia is observed. Pain Med. 2003;4:244-256.

34. Hayes S, Luoma JB, Bond FW, Masuda A, Lillis J. Acceptance and commitment therapy: model, process and outcomes. Behav Res Ther. 2006; 44:1-25.

35. Wilson KG, Groom J. Valued Living Questionnaire. Available from the first author at Department of Psychology, University of Mississippi, University, MS, 2002.

36. Wilson KG, Sandoz EK, Kitchens J. The Valued Living Questionnaire: defining and measuring valued action within a behavioral framework. Psychol Rec. 2010;60:249-272.

37. Keogh E, Bond FW, Hanmer R, Tilston J. Comparing acceptance- and control-based coping instructions on the cold-pressor pain experiences of healthy men and women. Eur J Pain. 2005;9:591-598.

38. Van Damme S, Legrain V, Vogt J, Crombez G. Keeping pain in mind: a motivational account of attention to pain. Neurosci Biobehav Rev. 2010;34:204-213. 
Journal of Pain Research

\section{Publish your work in this journal}

The Journal of Pain Research is an international, peer-reviewed, open access, online journal that welcomes laboratory and clinical findings in the fields of pain research and the prevention and management of pain. Original research, reviews, symposium reports, hypothesis formation and commentaries are all considered for publication.

The manuscript management system is completely online and includes a very quick and fair peer-review system, which is all easy to use. Visit http://www.dovepress.com/testimonials.php to read real quotes from published authors.

\footnotetext{
Submit your manuscript here: http://www.dovepress.com/journal-of-pain-research-journal
} 